\title{
Issues in the Influence of Ito-type Noise on the Oscillation of Solutions of Delay Differential Pantograph Equations
}

\author{
Augustine O. Atonuje \\ Department of Mathematics and Computer Science, Delta State University, Abraka, Nigeria.
}

Received: July 27, 2015 / Accepted: August 25, 2015 / Published: November 25, 2015.

\begin{abstract}
In this paper, a deterministic delay differential pantograph equation (DDPE) with an unbounded memory is stochastically perturbed by an Ito-type noise. The contribution of white noise to the oscillatory behaviour of the new stochastic delay differential pantograph equation (SDDPE) is investigated. It is established that under certain conditions and with a highly positive probability, the new stochastic delay differential pantograph equation has an oscillatory solution influenced by the presence of the noise. This is not possible with the original deterministic system which has a non-oscillatory solution due to the absence of noise.
\end{abstract}

Keywords: Delay differential pantograph equation, unbounded memory, Ito-type noise, oscillatory behaviour, stochastic delay differential pantograph equation.

\section{Introduction}

Towards the end of the $20^{\text {th }}$ century and early $21^{\text {st }}$ century, much of the research in the oscillatory theory of differential equations was related to an attempt by mathematicians to provide necessary and sufficient conditions for oscillation of solutions of deterministic delay differential equations. Following from the work of Li (1996) and Agwo (1999), Tang and Yu (2000) studied the oscillatory criteria for all solutions of the delay differential equation of the form:

$$
x^{\prime}(t)+p(t) x(r(t))=0, t \geq 0
$$

The authors established that if $r(t)$ is strictly increasing on $\left[t_{0}, \infty\right)$ with inverse $r^{-1}(t)$ and there exists an integer $k>0$ such that $r^{-k}(t)$ is defined on $\left[t_{0}, \infty\right)$ by

$$
r^{-1(k+1)}(t)=r^{-1}\left(r^{-k}(t)\right), \quad k=1,2,3, \ldots \ldots
$$

Corresponding author: Augustine O. Atonuje, Department of Mathematics and Computer Science, Delta State University, Abraka, Nigeria. E-mail: austino412@yahoo.com.
Suppose also that there exists an integer $n>0$ and $T_{0} \geq r^{-n}\left(t_{0}\right)$ such that

$$
p_{n}(t) \geq \frac{1}{e^{n}} \quad \text { and } \quad \bar{p}_{n}(t) \geq \frac{1}{e^{n}}, \quad t \geq T_{0}
$$

and

$$
\int_{T_{0}}^{\infty} p(t)\left[\exp \left(e^{n-1} p_{n}(t)-\frac{1}{e}\right)-1\right] d t=\infty
$$

where

$$
p_{1}(t)=\int_{r(t)}^{t} p(s) d s
$$

$$
p_{k+1}(t)=\int_{r(t)}^{t} p(s) p_{k}(s) d s, t \geq r^{-k-1}\left(t_{0}\right)
$$

and

$$
\begin{gathered}
\bar{p}_{1}(t)=\int_{t}^{r^{-1}(t)} p(s) d s \\
\bar{p}_{k+1}(t)=\int_{t}^{r^{-1}(t)} p(s) \bar{p}_{k}(s) d s
\end{gathered}
$$




$$
t \geq t_{0}, k=1,2, \ldots
$$

Then all solutions of eq. (1) oscillate.

For detailed understanding of results on oscillation of solutions of deterministic delay differential equations, the reader is referred to the interesting works of Ahmad (2003), Bainov et al (1998), Elabbasy et al (2000), Yu and Wang (1994), Gopalsamy (1987) and some of the references therein.

While the presence of time lag is often cited as a motivation for oscillation in the cases above, only recently has the effect of white noise or stochasticity on the oscillatory behaviour of dynamical systems been investigated. (See for example Appleby and Buckwar (2005), Appleby and Kelly (2004), Atonuje and Ayoola (2008), etc.). The motivation of this article is the desire to present a better understanding of the contribution of white noise of Ito-type to the oscillatory behaviour of solutions of a deterministic first order delay differential pantograph equation of the form

$$
\left.\begin{array}{c}
x^{\mid}(t)=a x(t)+b x(q t) \\
x(t)=\phi(t), t \in[-\Gamma, 0]
\end{array}\right\}
$$

By solution of Eq. (5), I mean a function $x(t) \in C\left([\underline{t}, 0), \mathfrak{R}^{+}\right), \underline{t} \geq t_{0}$, where $\underline{t}=\inf \left\{r(t): t \geq t_{0}\right\}$ which satisfies Eq.(5) together with its initial function for all $t \geq t_{0}$. Eq.(5) is stochastically perturbed by an Ito-type noise to obtain a stochastic delay differential pantograph equation of the form given by

$$
\left.\begin{array}{c}
X(t)=\{a X(t)+b X(q t)\} d t+ \\
\left\{\sigma_{1}+\sigma_{2} X(t)+\sigma_{3} X(q t)\right\} d B(t), t \geq 0 \\
x(t)=\varphi(t), t \in[-\bar{\Gamma}, 0]
\end{array}\right\}
$$

where $r(t) \leq \bar{\Gamma}$ is a continuous function called time lag or unbounded memory which satisfies the following conditions: $q t<t, t \geq 0, t \rightarrow t-q t$ is non-decreasing and $-\bar{\Gamma}=\inf _{t \geq 0} t-q t, q t \rightarrow \infty$, as $t \rightarrow \infty$, the initial function $\phi(t) \in C([(-\bar{\Gamma}, 0), \mathfrak{R}])$, a
Banach space of all continuous sample paths from $[-\bar{\Gamma}, 0]$ to $\mathfrak{R}$ equipped with the supremum norm $\quad\|\xi\|=\operatorname{Sup}_{s \in[-\bar{\Gamma}, 0]}|\xi(s)|$, where $\xi \in C \quad$ and $\{B(t)\}_{t \geq 0}$ is a one-dimensional Brownian motion defined on the probability triple $(\Omega, F, P)$ with filtration $\{F(t)\}_{t \geq 0}$ which is right continuous and contains all p-null sets in F; $\Omega=\left(\mathfrak{R}, \mathfrak{R}^{m}\right)$ is the set of continuous functions on $\mathfrak{R} ; \mathrm{F}$ is the $\sigma-$ algebra of Borel sets and $\mathrm{P}$ is the probability measure on $\mathrm{F}$; also $a, b, \sigma_{1}, \sigma_{2}, \sigma_{3} \in \mathfrak{R}^{+}$. Throughout the present paper, the oscillatory behaviour of the stochastic delay differential pantograph equation (6) would always be compared with the oscillatory behaviour of the deterministic delay differential equation (5)

The deterministic and the stochastic delay differential pantograph equations are applicable in many areas which include overheat current collection in electric locomotive, wave motion, stretched strings under gravity, active vibration and noise control systems, conveyor belts, metal rolling systems, electronic transmission lines, etc. For detail understanding of deterministic and stochastic delay differential pantograph equations, the reader is referred to the work of Baker and Buckwar (2000) which is referenced in this article.

A question of mathematical and biological interest is whether sustained oscillation is possible, with an almost certain probability for the SDDPE (6) even when the deterministic DDPE (5), where noise is absent, has an oscillatory solution under certain conditions on the noise intensity and for any initial datum. In the present paper, a detailed answer is provided to this question. It is expected that this article will stimulate other researchers in the desire to investigate the influence or input of white noise to the oscillatory behavior of solutions of stochastic delay differential equations. 


\section{Basic Results and Preliminaries}

Eq. (6) can be expressed as an integral equation of the form

$$
\begin{aligned}
& X(t)=X_{0}+\int_{0}^{t}\{a X(s)+b X(q t)\} d s+ \\
& \int_{0}^{t}\left\{\sigma_{1}+\sigma_{2} X(s)+\sigma_{3} X(q t)\right\} d B(s)
\end{aligned}
$$

Eq. (5) and Eq. (6) have the same initial function. To the best of the author's knowledge, the interplay between sufficiently small time delay and the presence of white noise as a motivating factor for oscillation of solutions of delay differential pantograph equations has not been previously investigated.

The key step to establishing sustained oscillation of solutions of the delay differential pantograph equation due to noise influence, involves the transformation of the solution of the SDDPE (6) into a conjugation relation between a continuously differentiable solution of a scalar random delay differential equation and a stationary coordinate process or change. This approach of formulating a stationary coordinate process and conjugation of flows for stochastic differential equations is the subject of the beautiful works of Lisei (2001) and Kunita (1997). A paper on an alternative method of decomposition of solutions of SDDEs into a product of generic random process and a solution of a random scalar delay differential equation was written by Appleby and Buckwar (2005).

Recall that a measurable sample - continuous real-valued stochastic process $X(t)$ defined on a probability space $(\Omega, F, P)$ is said to be a strong solution of SDDPE (6) if it is $\left\{F_{t}\right\}_{t \geq 0}$-adapted and satisfies Eq.(6) as well as its initial condition. By uniqueness of $X(t)$, the author refers to the existence of any other solution $Y(t)$ of the equation which is indistinguishable from $X(t)$ in the sense that

$$
P(X(t)=Y(t), t \in[-\Gamma, 0])=1 .
$$

\section{Definition 1:}

A solution $x(t)$ of the DDE (5) defined on the time horizon $\left[T_{x}, \infty\right)$ which satisfies $\operatorname{Sup}\{|x(t)|: t>T\}>0$ for every $T \geq T_{X}$, that is $|x(t)| \neq 0$, in any infinite interval $\left[T_{x}, \infty\right)$ is called a regular or non-trivial solution.

A trivial solution $x(t)$ of Eq. (5) is said to be eventually positive if there exists $t_{1}>0$ such that $x(t)>0$ for $t>t_{1}$. A trivial solution $x(t)$ to the DDE is said to be eventually negative if there exists $t_{1}>0$ such that $x(t)<0$, for $t \geq t_{1}$.

\section{Definition 2:}

A non- trivial solution $x(t)$ of the DDE (5) is said to be oscillatory if and only if it has arbitrarily large (many) zeros for $t>0$. That is, there exists a sequence $\left\{t_{n}: x\left(t_{n}\right)=0\right\} \quad$ of $x(t)$ such that $\operatorname{Limit}_{n \rightarrow \infty}=+\infty$, otherwise, it is said to be non-oscillatory. $x(t)$ is non-oscillatory if there exists $t_{1}>0$ such that $x(t) \neq 0$ for $t \geq t_{1}$. A non-oscillatory solution is either eventually positive or eventually negative.

For the stochastic delay differential pantograph equation, the solution $\{X(t, w)\}_{t \geq 0}$ defined on a probability space $(\Omega, F, P)$ with continuous sample path is said to be almost certainly oscillatory if there exists $\Omega^{*} \subseteq \Omega$ with $P\left(\Omega^{*}\right)=1$ such that for all $w \in \Omega^{*}$, the path $X(., w)$ is oscillatory. Otherwise it is said to be non-oscillatory.

The study involves expressing the solution of the SDDPE (6) into a conjugation relation between the solution of a random scalar delay differential equation and a stationary coordinate change or process.

\section{The Random Delay Equation and Conjugation Relation:}

Consider the following scalar delay differential equation

$$
Z^{\mid}(t)=Z_{0}-\int_{0}^{t} K(t) Z(q s) d s \quad t \geq 0
$$


For all $q t \in \mathfrak{R}, w \in \Omega$, Eq. (8) admits a continuously differentiable solution $\{Z(t, w)\}_{t \geq 0}$ such that $t \rightarrow Z(t, w)$ is continuous for all $w \in \Omega$, where $q t$ is an unbounded memory and $K($.$) is a$ random continuous non-negative function defined on some subset $\Omega^{*} \subseteq \Omega$ by

$$
K(t)=\left\{\begin{array}{c}
-b \exp (-\lambda q t) \exp (-\mu(B(t, w))-B(t-q t)(w)), \\
\quad \text { for } t>t \quad \exp (-\lambda t-\mu B(t)(w)), \\
\quad \text { for } t<\underline{t}
\end{array}\right.
$$

where $\lambda=\left(a-\frac{\mu^{2}}{2}\right), \underline{t}=\inf (t>0: t-q t)$ such that for all $t>\underline{t}, t-q t \geq 0$ and $w \in \Omega$. We see that $\mathrm{K}$ depends upon the increments of a standard Brownian motion $\{B(t)\}_{t \geq 0}$. The deviations in these increments ensure that $\mathrm{K}$ is large enough to stimulate oscillation in the solution of Eq. (8). Equation (8) admits a solution $\{Z(t, w)\}_{t \geq 0}$ which is continuously differentiable.

Assume that $q t$ is bounded such that for $0<q t<\infty \quad t \rightarrow t-q t$ is non-decreasing and introduce a random coordinate change $\{\gamma(t, .)\}_{t \geq 0}$ which enables the solution of the SDDPE (6) to be transformed into a conjugation relation with the solution $\{Z(t, w)\}_{t \geq 0}$ of Eq. (8).

\section{Lemma 1:}

Assume that $t \in \mathfrak{R}, q t=t, w \in \Omega$. Then the following properties hold for $\{\gamma(t, .)\}_{t \in \Re}$ :

$$
\{\gamma(t, .)\}_{t \geq 0} \text {. is a continuous } C^{k+1, \epsilon} \text { semi - }
$$
martingale for $0<\epsilon<\delta$ such that for all $w \in \Omega$, $w \rightarrow \gamma(t, w) \in \mathfrak{R}^{m}$ is a $C^{k+1}$ diffeomorphism of $\mathfrak{R}^{m}$. for all $t>s, v \in \mathfrak{R}^{m}, w \in \Omega$, there exists $\{\Lambda(t, .)\}_{t \in \Re}$ such that

$$
\begin{aligned}
& \gamma(t, v)=\gamma(s, v)+ \\
& \int_{s}^{t} N(d v, \gamma(u, v)) d u+\int_{s}^{t} \Lambda(u, v) d u
\end{aligned}
$$

The processes $\{\gamma(t, .)\}_{t \in \Re}$ and $\{\Lambda(t, .)\}_{t \in \Re}$ are perfectly stationary such that

$$
\begin{aligned}
& \gamma(t, v, w)=\gamma(0, v, \theta(t, w)) \\
& \Lambda(t, v, w)=\Lambda(0, v, \theta(t, w)) \\
& \text { for all } t \in \mathfrak{R}, v \in \mathfrak{R}, w \in \Omega
\end{aligned}
$$

\section{Proposition 1:}

Assume that $\{\mathrm{X}(\mathrm{t}, \mathrm{w})\}_{t \geq 0}$ is the solution of the stochastic delay differential pantograph equation

$$
\begin{aligned}
& X(t)=X_{0}+\int_{0}^{t}\{a X(s)+b X(q t)\} d s \\
& +\int_{0}^{t}\left\{\sigma_{1}+\sigma_{2} X(s)+\sigma_{3} X(q t)\right\} d B(s)
\end{aligned}
$$

Let $\{Z(t, w)\}_{t \geq 0}$ be the solution of the random scalar delay differential equation (8). Then the following conjugation relation holds:

$X(t, w)=\gamma(0, . ., \theta(t, w)) \circ Z(t, w) \circ \gamma^{-1}(0, ., \theta(t, w))$, $t \geq 0, w \in \Omega$

It is well known that deterministic delay differential equations usually possess oscillatory solutions if and only if their characteristic equations have no real roots.

By putting $x(t)=z(t) e^{a t}$ in the deterministic DDPE (5), one sees that

$$
\begin{aligned}
& x^{\prime}(t)=e^{a t} z^{\mid}(t)+a e^{a t} z(t) \\
& x(q t)=z(q t) e^{a(q t)}
\end{aligned}
$$

Substituting in Eq. (5), one gets

$$
\begin{aligned}
& e^{a t} z^{\prime}(t)+a e^{a t} z(t)=a z(t) e^{a t}+b z(q t) e^{a(q t)} \\
& z^{\mid}(t)=b e^{-a t} z(q t) e^{a(q t)} \\
& =b e^{a(q t-t)} z(q t) \\
& z^{\prime}(t)=b e^{-a(t-q t)} z(q t)
\end{aligned}
$$

Eq.(12) is the characteristic or pure delay equation of the classical DDPE (5). Useful results of the rich oscillation theory of classical differential equations can now be recalled for use to investigate the influence of Ito-type noise on the oscillatory behavior of the stochastic delay differential pantograph 
equation. The reader can find some of these most extensive results in the works of Driver (1977), Gyori and Ladas (1991) and Shevelo (1978). For application in the present paper, the following is a special case of the results on oscillatory criteria which can be found in Elbert and Stavroulakis (1995) (Theorem 2.16) and specifically in Ladde et al (1987) (Theorem 2.1.1).

\section{Proposition 1:}

Assume that $q t$ is strictly increasing on $\left[t_{0}, \infty\right)$ and that $p(t) \in A_{\lambda}$ for some $\lambda \in(0,1)$ and either

$$
\lambda \underset{n \rightarrow \infty}{\operatorname{LimSup}} n \sum_{i=n}^{\infty}\left(\int_{t_{i-1}}^{t_{i}} p(s) d s-\frac{1}{e}\right)>\frac{2}{e}
$$

or

$$
\lambda \underset{n \rightarrow \infty}{\operatorname{Liminf}} n \sum_{i=n}^{\infty}\left(\int_{t_{i-1}}^{t_{i}} p(s) d s-\frac{1}{e}\right)>\frac{1}{2 e}
$$

or

$$
\text { if } \operatorname{Liminf}_{t \rightarrow \infty} \int_{q t}^{t} p(s) d s>\frac{1}{e}
$$

Then all solutions of

$$
x^{l}(t)=-p(s) x(q t) t \geq t_{0}
$$

oscillate.

For non-oscillatory results concerning Eq. (16), we have the following from $\mathrm{Yu}$ and Tang (2001), (Theorem 2.18).

\section{Proposition 2:}

Let the piecewise continuous function $p:\left[t_{0}, \infty\right) \rightarrow[0, \infty)$ belong to $A_{\lambda}$. If

$$
\int_{q t}^{t} p(s) d s \geq \frac{1}{e}
$$

for sufficiently large $t$ and

$$
\underset{t \rightarrow \infty}{\operatorname{LimSup}}\left[\left(\int_{q t}^{t} p(s) d s-\frac{1}{e}\right)\left(\int_{t_{0}}^{t} p(s) d s\right)^{2}\right]<\frac{1}{8 e^{3}}
$$

Then Eq. (16) has an eventually positive solution and hence non-oscillatory.

\section{Sustained Oscillation Influenced By Stochasticity}

In this section, we study the oscillatory behavior of the stochastic delay pantograph equation (6). It is established that if the drift coefficient is sufficiently large under certain conditions on time lag, the SDDPE can possess an eventually positive solution and hence non-oscillatory. On the other hand, if the drift is small and the time lag $q t$ decays quite rapidly for any initial datum, then all solutions of Eq. (6) are almost certainly oscillatory. The analysis of the sustained oscillation in the solution of the SDDPE is possible by contrasting the oscillatory results with those of the corresponding deterministic DDPE (5).

The following Lemmas are needed for the main results:

\section{Lemma 1:}

Assume that $\operatorname{LimSup}_{t \rightarrow \infty} \int_{t}^{q t} p(s) d s>0$ and $x(t)$ is an eventually positive solution of Eq. (16), then

$$
\operatorname{Liminf}_{t \rightarrow \infty} \frac{x(q t)}{x(t)}<\infty
$$

\section{Lemma 2:}

Assume that Eq. (16) has an eventually positive solution, then

$$
\int_{t}^{q t} p(s) d s \leq 1
$$

In the following proposition, it is established that whenever the drift coefficient is sufficiently large for some positive initial function, the SDDPE (6) can possess an eventually positive solution and hence non-oscillatory.

\section{Proposition 3:}

Assume that the initial function is positive, that is $\phi(t)>0, t \in[-\Gamma, 0]$, if $b>0$, then the SDDPE (6) has a non- oscillatory solution on the half plane $[0, \infty)$.

\section{Proof:}

Assume that the process $Z(., w)$ which solves the random scalar delay differential equation (8) satisfies the conjugation relation

$$
X(t, w)=\gamma(0, ., \theta(t, w)) \circ Z(t, w) \circ \gamma^{-1}(0,0, w)
$$

in the P-almost sure event $\Omega^{*} \subset \Omega$, for $w \in \Omega^{*}$. 
Define $t_{\otimes}(w)=\inf \{Z(t, w)=0: t \geq 0\}$ which is possible because $Z(t, w)-\phi(t) \geq 0, t \in[-\Gamma, 0]$ and as such $t_{\otimes}(w)>0$. Now, $Z^{1}((t, w), w)<0$. Moreover, $q t>0$ and hence one gets

$$
Z\left(t_{\otimes}(w)-q t_{\otimes}(w), w\right)>0 \text { as } Z(t, w)>0,
$$

for $t<t_{\otimes}(w)$ Since the stationary coordinate change $\gamma>0$ and the drift coefficient is also positive it follows from the relation Eq. (11) that $Z^{1}\left(t_{\otimes}(w), w\right)>0$ which contradicts the earlier assertion that $Z^{1}\left(t_{\otimes}(w), w\right)<0$.

Hence $Z(t, w)>0$, for all $t \geq 0$ which follows from Eq.(11) that the path $X(t, w)>0$ for all $t \geq 0$ and as such the solution $X(t)$ of the SDDPE (6) is eventually positive and hence non-oscillatory.

\section{Theorem 1:}

Assume that conditions $A_{1}-A$ hold and $\sigma_{1}=\sigma_{2}=0, \sigma_{3} \neq 0$. Then for any initial datum, if $b<0$, all solutions of Eq. (6) oscillate P-almost surely $0 \mathrm{n}[0, \infty)$.

\section{Proof:}

Using the conjugation relation

$$
X(t, w)=\gamma(0, ., \theta(t, w)) \circ Z(t, w) \circ \gamma^{-1}(0,0, w)
$$

one sees that the collection $Y=\{X(t)=0: t \geq 0\}$ satisfies $\operatorname{Sup} Y=+\infty$ P-almost surely. For, $t \geq 0, w \in \Omega$, introduce

$$
K(t, w)=-b \gamma(t-q t, w) \gamma^{-1}(t, w) .
$$

It follows that $K($.$) is an eventually positive$ function and hence $\mathrm{Z}$ satisfies

$$
Z^{1}(t, w)=-K(t, w) Z(t-q t, w), t \geq 0
$$

Now assume that there exists $\Omega^{*} \subset \Omega$ such that $q t$ is strictly increasing on $[0, \infty)$ and $K(t) \in A_{\lambda}$ for some $\lambda \in(0,1)$ such that

$$
\Omega^{*}=\left\{w \in \Omega: \lambda \operatorname{LimSup} n \sum_{i=n}^{\infty}\left(\int_{t_{i-1}}^{t_{i}} K(s) d s-\frac{1}{e}\right)>\frac{2}{e}, \operatorname{Liminf}_{t \rightarrow \infty} \int_{q t}^{t} K(s) d s>\frac{1}{2 e}\right\}, P\left[\Omega^{*}\right]=1
$$

It follows that $\mathrm{K}$ and $t-q t$ satisfy the condition of Proposition 1 for each $w \in \Omega^{*}$ and as such, the path $Z(., w)$ is oscillatory. If not, we set $\delta(t)=\max (q s)=s \in[0, t]$. It follows that

$$
\operatorname{Liminf}_{t \rightarrow \infty} \int_{\delta(t)}^{t} K(s) d s>\frac{1}{e}
$$

Assume that $Z(t)$ is an eventually positive solution of

$$
Z^{1}(t)+K(t) Z(q t) \leq 0
$$

such that $Z(q t)>0$ for $t>t_{1}$. By Eq. (18), there exists $t_{2} \geq t_{1}$ such that

$$
\int_{q t}^{t} K(s) d s \geq c>e^{-1} \text { for } t \geq t_{2}
$$

Dividing Eq. (19) by $Z(t)$ and integrating from qt to $t$, one gets

$$
\operatorname{In} \frac{Z(t)}{Z(q t)}+\int_{q t}^{t} K(s) d s \leq 0, t \geq t_{2}
$$

which follows that

$$
\operatorname{In} \frac{Z(q t)}{Z(t)} \geq \int_{q t}^{t} K(s) d s \geq c, t \geq t_{2}
$$

The following is true of exponential functions: $e^{x} \geq e x$, for some $x \geq 0$. Applying this to Eq. (21), it follows that

$$
\frac{Z(q t)}{Z(t)} \geq e c, t \geq t_{2}
$$

By iteration, there exists a sequence $\left\{t_{j}\right\}$ such that

$$
\frac{Z(q t)}{Z(t)} \geq(e c)^{k}, t \geq t_{j}
$$


From Eq. (20), there exists $t_{\otimes}$ such that

$$
\int_{q t}^{t_{\circledast}} K(s) d s \geq \frac{c}{2} \text { and } \int_{t_{\otimes}}^{t} K(s) d s \geq \frac{c}{2}, \text { for } t \geq t_{j}
$$

Integrating Eq. (19) from $q t$ to $t_{\otimes}$, one gets

$$
Z\left(t_{\otimes}\right)+Z(q t)+\int_{q t}^{t} K(s) Z(q t) d s \leq 0
$$

It follows that

$$
Z(q t) \geq Z\left(q t_{\otimes}\right) \frac{c}{2}
$$

In the same way, one gets

$$
Z(t)-Z\left(t_{\otimes}\right)+\int_{t_{\circledast}}^{t} K(s) Z(q s) d s \leq 0
$$

and as such

$$
Z\left(t_{\otimes}\right) \geq Z(q t) \frac{c}{2}
$$

Combining Eq. (23) and Eq. (24) one obtains the inequality

$$
Z\left(t_{\otimes}\right) \geq Z\left(q t_{\otimes}\right)\left(\frac{c}{2}\right)^{2}
$$

From Eq. (22) and Eq. (25), it follows that

$$
\left(\frac{2}{c}\right)^{2} \geq \frac{Z\left(q t_{\otimes}\right)}{Z\left(t_{\otimes}\right)} \geq(e c)^{j} \text { for all } t \geq t_{j}
$$

Since $e c>1$, one can choose $j$ sufficiently large such that

$$
(e c)^{j}>\left(\frac{2}{c}\right)^{2}
$$

Hence Eq. (26) is a contradiction. It therefore follows that the path $Z(., w)$ is oscillatory and from the conjugation relation in Eq.(11), it follows that the path $X(., w)$ is oscillatory. However, since $\Omega^{*}$ is a P-almost sure event, it follows that the solution $X(t)$ of Eq. (6) is P-almost surely oscillatory. One sees that

$$
\begin{aligned}
\int_{t-q t}^{t} K(s) d s & =\int_{t-q t}^{t}-b \exp \left(-\left(a-\frac{\sigma_{3}^{2}}{2}\right) q s\right) \exp \left(-\sigma_{3}(B(s)-B(s-q s))\right) d s \\
& \geq-b \max \left(1, \exp \left(-a-\frac{\sigma_{3}^{2}}{2}\right) \bar{\Gamma}\right) \int_{t-q t}^{t} \exp \left(-\sigma_{3}(B(s)-B(s-q s))\right) d s
\end{aligned}
$$

Suppose that

$$
\underset{t \rightarrow \infty}{\operatorname{LimSup}} \int_{t-q t}^{t} \exp \left(-\sigma_{3}(B(s)-B(s-q s))\right) d s=\infty
$$

then the event $\Omega^{*} \subset \Omega$ exists P-almost surely and hence the result.

\section{Discussion:}

The conjugation relation in Eq.(11) relates the solution $X(t)$ of the SDDPE (6) with the continuously differentiable function $Z(t)$ which solves the scalar DDE (8) via the stationary coordinate change or process whose properties are well outlined. Using these relations and some results from the oscillatory theory of classical differential equations, it is now possible to acquire information about the oscillatory behavior of the solution path $X(t, w)$ of the SDDPE (6).

The Brownian increment in Eq. (28) is the main factor which sustains oscillation in the SDDPE (6). In contrast, the deterministic Eq.(5) where $\sigma_{1}=\sigma_{2}=\sigma_{3}=0$, the absence of noise perturbation ensures that the condition of Proposition 2 is satisfied and at that point, the non-stochastic Eq. (5), where noise is absent can have a non-oscillatory solution P-almost surely. It is not possible for the SDDPE to have a non-oscillatory solution since Eq. (28) must always hold due to the presence of noise which ensures that the conditions of Proposition 1 hold.

\section{References}

[1] Li, B. (1996), Oscillation of first order delay differential 


\section{Delay Differential Pantograph Equations}

equations. Proc. Amer. Math. Soc. Vol. 124, N0. 12, 3729-3737.

[2] Agwo, H (1999). On the oscillation of delay differential equations with real coefficient. Int. Jour. Math \& Math. Sci. Vol. 22, No. 3573-578.

[3] Tang, X. H. and Yu, J. S.(2000), First order linear delay differential inequalities. ACTA. Math. Scientia, Vol. 20, N0. 2, 269-273.

[4] Ahmad, F. (2003). Linear differential equation with a positive and negative term. Elect. Jour. Diff. Equ. Vol.2OO3, No 92, pp 1-6.

[5] Bainov, D. D; Dimitrova, M. B. and Simeonov, P. S. (1994), Oscillatory properties of the solutions of impulsive differential equations with retarded argument and oscillating coefficients. Jour. Appl. Anal. Vol. 4, N0. 1, 63-74.

[6] Elabbasy, E. M; Hegazi, A. S. and Saker, S. H. (2000), Oscillation of solutions to delay differential equations with positive and negative coefficients. Elect. Jour. Diff. Equ. Vol. 2000, No. 13, 1-13.

[7] Yu, J. S. and Wang Z. (1994), Oscillation and existence of positive solutions in differential equations. Soochow Jour. Math. Vol. 20, N0. 2, 193-204.

[8] Gopalsamy, K. (1987), Oscillatory properties of systems of first order linear delay differential inequalities. Pacific Jour. Math. Vol. 128, N0. 2, 299-305.

[9] Appleby, J. A. D. and Buckwar, E. (2005). Noise induced oscillation in solutions of delay differential equations. Dynamic Systems and Applications. 14(2) 175-196.

[10] Appleby, J. A. D. and Kelly, C. (2004). Asymptotic and oscillatory properties of linear stochastic delay differential equations with vanishing delay. Funct. Diff. Equation, Vol. 11, (3-4), 235-265.

[11] Atonuje, A. O. and Ayoola, E. O. (2007). On the complementary roles of noise and delay in the oscillatory behavior of stochastic delay differential equations, Jour. of Math. Sci. 19, No.1, 11-20.

[12] Baker, C. T. H. and Buckwar, E. (2000), Continuous $\theta$-Methods for the stochastic pantograph equation. Electronic Transactions On Numerical Analysis, Vol. 11, 131-151.

[13] Lisei, H. (2001), Conjugation of flows for stochastic and random functional differential equation. Stochastic and Dynamics, Vol. 1, N0. 2, 283 - 298.

[14] Kunita, H. (1997), Stochastic flows and stochastic differential equations. Cambridge University Press.

[15] Driver, R. D. (1977), Ordinary and delay differential equations. Appl. Math Sci. 20 Springer, New York.

[16] Gyori, I. and Ladas, G.(1991), Oscillation theory of delay differential equations with applications. Clarendon Press, Oxford.

[17] Shevelo, V. N. (1978), Oscillations of solutions of differential equations with deviating argument. Naukova Dumka, Kiev. (In Russia)

[18] Elbert, A. and Stavroulakis, I. P. (1995), Oscillation and non-oscillation criteria for delay differential equations. Proc. Amer. Math, Soc. 123, 1503-1510.

[19] Ladas, G. S; Laskhmikanthan, V. and Zhang, B. G. (1987), Theory of differential equations with deviating argument. Marcel Dekker, New York.

[20] Yu, J. S. and Tang, X. H. (2001), Comparison theorems in delay differential equations in a critical state and application. Proc. London Math Soc. 63, 188-204. 